\title{
Sharpening some core theorems of Nieto and Rodríguez-López with application to boundary value problem
}

\author{
Marwan Amin Kutbi ${ }^{1 *}$, Aftab Alam² and Mohammad Imdad ${ }^{2}$
}

"Correspondence:

mhimdad@gmail.com

${ }^{2}$ Department of Mathematics,

Aligarh Muslim University, Aligarh,

Uttar Pradesh 202002, India

Full list of author information is

available at the end of the article

\section{Springer}

\begin{abstract}
In this paper, we prove sharpened versions of some classical order-theoretic metrical fixed point theorems due to Nieto and Rodríguez-López (Order 22(3):223-239, 2005) using order-theoretic variants of completeness and continuity besides some another notions such as: the ICC property, the DCC property, and the MCC property. In this continuation, we further extend our results for Boyd-Wong type nonlinear contractions. Finally, as an application of our certain newly proved results, we establish the existence and uniqueness of solution of a first order periodic boundary value problem.
\end{abstract}

MSC: $47 \mathrm{H} 10 ; 54 \mathrm{H} 25 ; 34 \mathrm{~B} 15$

Keywords: ordered metric space; O-completeness; O-continuity; MCC property

\section{Introduction}

Throughout this paper, the pair $(X, \preceq)$, stands for a nonempty set $X$ equipped with a partial order $\preceq$ often called an ordered set wherein $x \succeq y$ means $y \preceq x$. Two elements $x$ and $y$ in an ordered set $(X, \preceq)$ are said to be comparable if either $x \preceq y$ or $x \succeq y$ and denote it by $x \prec \succ y$. A subset $E$ of an ordered set $(X, \preceq)$ is called totally ordered if $x \prec \succ y$ for all $x, y \in E$. A self-mapping $f$ defined on an ordered set $(X, \preceq)$ is called increasing (or isotone or order-preserving) if for any $x, y \in X, x \preceq y$ implies $f(x) \preceq f(y)$. As per standard practice, we can define the notions of increasing, decreasing, monotone, bounded above and bounded below sequences besides bounds (upper as well as lower) of a sequence in an ordered set $(X, \preceq)$, which on the set of real numbers under natural ordering coincide with their usual senses. Following O'Regan and Petruşel [1], the triple $(X, d, \preceq)$ is called ordered metric space wherein $X$ denotes a nonempty set endowed with a metric $d$ and a partial order $\preceq$. If in addition, $d$ is a complete metric on $X$, then we say that $(X, d, \preceq)$ is an ordered complete metric space.

In the recent years, a multitude of order-theoretic metrical fixed point theorems have been proved for order-preserving contractions. In such results, the involved contraction condition is considerably weakened as one is merely required to hold only on those elements which are comparable in the underlying partial ordering. The techniques involved in the proofs of such results is the combination of ideas used in the contraction principle together with the one employed in monotone iterative technique. This trend is essentially

(c) 2015 Kutbi et al. This article is distributed under the terms of the Creative Commons Attribution 4.0 International License (http://creativecommons.org/licenses/by/4.0/), which permits unrestricted use, distribution, and reproduction in any medium, provided you give appropriate credit to the original author(s) and the source, provide a link to the Creative Commons license, and indicate if changes were made. 
initiated by Turinici [2,3]. Later, Ran and Reurings [4] proved a slightly more natural version of the corresponding fixed point theorems of Turinici $(c f .[2,3])$ for continuous monotone mappings with some applications to matrix equations. In the same lieu, Nieto and Rodríguez-López [5] proved some variants of Ran and Reuring fixed point theorem for increasing mappings. Nieto and Rodríguez-López fixed point theorems were also extended by several authors (see [1, 6-10]).

Before discussing such results, we summarize some relevant basic terminologies needed in our subsequent discussion. Throughout this manuscript, $\mathbb{N}$ stands for the set of natural numbers and $\mathbb{N}_{0}$ for the set of whole numbers (i.e. $\mathbb{N}_{0}=\mathbb{N} \cup\{0\}$ ).

Let $(X, d, \preceq)$ be an ordered metric space and $\left\{x_{n}\right\}$ a sequence in $X$. We adopt the following notations.

(i) If $\left\{x_{n}\right\}$ is increasing and $x_{n} \stackrel{d}{\longrightarrow} x$, then we denote it symbolically by $x_{n} \uparrow x$.

(ii) If $\left\{x_{n}\right\}$ is decreasing and $x_{n} \stackrel{d}{\longrightarrow} x$, then we denote it symbolically by $x_{n} \downarrow x$.

(iii) If $\left\{x_{n}\right\}$ is monotone and $x_{n} \stackrel{d}{\longrightarrow} x$, then we denote it symbolically by $x_{n} \uparrow \downarrow x$.

Alam et al. [9] formulated the following notions by using certain properties on ordered metric space (in order to avoid the necessity of continuity requirement on underlying mapping) utilized by earlier authors especially from $[5,11]$ besides some other ones.

Definition $1[9]$ Let $(X, d, \preceq)$ be an ordered metric space. We say that

(i) $(X, d, \preceq)$ has the ICU (increasing-convergence-upper bound) property if every increasing convergent sequence $\left\{x_{n}\right\}$ in $X$ is bounded above by its limit (as an upper bound), i.e.,

$$
x_{n} \uparrow x \Rightarrow x_{n} \preceq x \quad \forall n \in \mathbb{N}_{0},
$$

(ii) $(X, d, \preceq$ ) has the $D C L$ (decreasing-convergence-lower bound) property if every decreasing convergent sequence $\left\{x_{n}\right\}$ in $X$ is bounded below by its limit (as a lower bound), i.e.,

$$
x_{n} \downarrow x \quad \Rightarrow \quad x_{n} \succeq x \quad \forall n \in \mathbb{N}_{0} \text {, and }
$$

(iii) $(X, d, \preceq$ ) has the $M C B$ (monotone-convergence-boundedness) property if $X$ has both the ICU and the $D C L$ property.

Alam et al. [10] further weakened the notions embodied in Definition 1 as follows.

Definition 2 [10] Let $(X, d, \preceq)$ be an ordered metric space. We say that

(i) $(X, d, \preceq)$ has the ICC (increasing-convergence-comparable) property if every increasing convergent sequence $\left\{x_{n}\right\}$ in $X$ has a subsequence $\left\{x_{n_{k}}\right\}$ such that every term of $\left\{x_{n_{k}}\right\}$ is comparable with the limit of $\left\{x_{n}\right\}$, i.e.,

$$
x_{n} \uparrow x \Rightarrow \exists \text { a subsequence }\left\{x_{n_{k}}\right\} \text { of }\left\{x_{n}\right\} \text { with } x_{n_{k}} \prec \succ x \quad \forall k \in \mathbb{N}_{0},
$$

(ii) $(X, d, \preceq)$ has the $D C C$ (decreasing-convergence-comparable) property if every decreasing convergent sequence $\left\{x_{n}\right\}$ in $X$ has a subsequence $\left\{x_{n_{k}}\right\}$ such that every 
term of $\left\{x_{n_{k}}\right\}$ is comparable with the limit of $\left\{x_{n}\right\}$, i.e.,

$$
x_{n} \downarrow x \Rightarrow \exists \text { a subsequence }\left\{x_{n_{k}}\right\} \text { of }\left\{x_{n}\right\} \text { with } x_{n_{k}} \prec \succ x \quad \forall k \in \mathbb{N}_{0} \text {, and }
$$

(iii) $(X, d, \preceq$ ) has the $M C C$ (monotone-convergence-comparable) property if every monotone convergent sequence $\left\{x_{n}\right\}$ in $X$ has a subsequence $\left\{x_{n_{k}}\right\}$ such that every term of $\left\{x_{n_{k}}\right\}$ is comparable with the limit of $\left\{x_{n}\right\}$, i.e.,

$$
x_{n} \uparrow \downarrow x \Rightarrow \exists \text { a subsequence }\left\{x_{n_{k}}\right\} \text { of }\left\{x_{n}\right\} \text { with } x_{n_{k}} \prec \succ x \quad \forall k \in \mathbb{N}_{0} \text {. }
$$

Remark 1 For an ordered metric space:

$I C U$ property $\Rightarrow I C C$ property.

$D C L$ property $\Rightarrow D C C$ property.

$M C B$ property $\Rightarrow M C C$ property $\Rightarrow I C C$ property as well as $D C C$ property.

Jleli et al. [12] formulated the following notion by using a certain property on an ordered set (in order to prove the uniqueness of fixed points) utilized by Nieto and RodríguezLópez [5].

Definition 3 [12] Let $(X, \preceq)$ be an ordered set and $f$ a self-mappings on $X$. We say that $(X, \preceq)$ is directed if for each pair $x, y \in X, \exists z \in X$ such that $x \prec \succ z$ and $y \prec \succ z$.

Notice that $(X, \preceq)$ is directed if and only if every pair of elements of $X$ has a lower bound or an upper bound (cf. [5]).

Definition 4 [13] Let $(X, \preceq)$ be an ordered set and $x, y \in X$. A finite subset $\left\{z_{1}, z_{2}, \ldots, z_{k}\right\} \subseteq$ $X$ is called $\prec \succ$-chain between $x$ and $y$ if

(i) $k \geq 2$,

(ii) $z_{1}=x$ and $z_{k}=y$,

(iii) $z_{1} \prec \succ z_{2} \prec \succ \cdots \prec \succ z_{k-1} \prec \succ z_{k}$.

We denote by $\mathrm{C}(x, y, \prec \succ)$ the class of all $\prec \succ$-chains between $x$ and $y$. If $(X, \preceq)$ is directed then $C(x, y, \prec \succ)$ is nonempty, for each $x, y \in X(c f$. [13]).

For the sake of completeness, we record the following well-known core results.

Theorem 1 (Nieto and Rodríguez-López [5]) Let $(X, d, \preceq)$ be an ordered metric space and $f$ a self-mapping on $X$. Suppose that the following conditions hold:

(a) $(X, d)$ is complete,

(b) $f$ is increasing,

(c) either $f$ is continuous or $(X, d, \preceq)$ has the ICU property,

(d) there exists $x_{0} \in X$ such that $x_{0} \preceq f\left(x_{0}\right)$,

(e) there exists $\alpha \in[0,1)$ such that

$$
d(f x, f y) \leq \alpha d(x, y) \quad \forall x, y \in X \text { with } x \prec \succ y .
$$

Then $f$ has a fixed point. 
Theorem 2 (Nieto and Rodríguez-López [5]) Theorem 1 remains true if the conditions (c) and $(\mathrm{d})$ are replaced by the conditions $(\mathrm{c})^{\prime}$ and $(\mathrm{d})^{\prime}$, respectively (besides retaining the rest of the hypotheses):

(c)' either $f$ is continuous or $(X, d, \preceq)$ has the DCL property,

(d)' there exists $x_{0} \in X$ such that $x_{0} \succeq f\left(x_{0}\right)$.

Theorem 3 (Nieto and Rodríguez-López [5]) In addition to the hypotheses of Theorem 1 (resp. Theorem 2), suppose that the following condition holds:

(f) $(X, \preceq)$ is directed.

Then $f$ has a unique fixed point.

Theorem 4 (Turinici [13]) Theorem 3 remains true if the condition ( $\mathrm{f}$ ) is replaced by the following condition (besides retaining the rest of the hypotheses):

(f) $)^{\prime} \mathrm{C}(x, y, \prec \succ)$ is nonempty for each $x, y \in X$.

Recently, Alam et al. [10] adopted the notions of completeness and continuity with respect to order-theoretic metrical structure, which run as follows.

Definition 5 [10] An ordered metric space $(X, d, \preceq)$ is called

(i) $\overline{\mathrm{O}}$-complete if every increasing Cauchy sequence in $X$ converges,

(ii) O-complete if every decreasing Cauchy sequence in $X$ converges, and

(iii) O-complete if every monotone Cauchy sequence in $X$ converges.

Here it can be pointed out that the notion of $\overline{\mathrm{O}}$-completeness is previously defined by Turinici [14] by recalling that $d$ is $(\preceq)$-complete.

Remark 2 In an ordered metric space, completeness $\Rightarrow$ O-completeness $\Rightarrow \overline{\mathrm{O}}$-completeness as well as $\underline{\mathrm{O}}$-completeness.

Definition 6 [10] Let $(X, d, \preceq)$ be an ordered metric space, $f: X \rightarrow X$ a mapping and $x \in X$. Then $f$ is called

(i) $\overline{\mathrm{O}}$-continuous at $x \in X$ if for any sequence $\left\{x_{n}\right\} \subset X$,

$$
x_{n} \uparrow x \Rightarrow f\left(x_{n}\right) \stackrel{d}{\longrightarrow} f(x),
$$

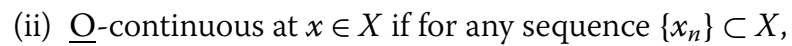

$$
x_{n} \downarrow x \Rightarrow f\left(x_{n}\right) \stackrel{d}{\longrightarrow} f(x) \text {, and }
$$

(iii) O-continuous at $x \in X$ if for any sequence $\left\{x_{n}\right\} \subset X$,

$$
x_{n} \uparrow \downarrow x \Rightarrow f\left(x_{n}\right) \stackrel{d}{\longrightarrow} f(x) .
$$

Moreover, $f$ is called $\mathrm{O}$-continuous (resp. $\overline{\mathrm{O}}$-continuous, $\underline{\mathrm{O}}$-continuous) if it is $\mathrm{O}$ -

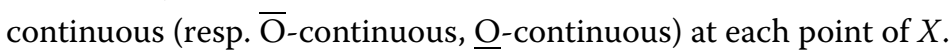


Here it can be pointed out that the notion of $\overline{\mathrm{O}}$-continuity is previously defined by Turinici [14] by recalling that $f$ is $(d, \preceq)$-continuous.

Remark 3 In an ordered metric space, continuity $\Rightarrow$ O-continuity $\Rightarrow \overline{\mathrm{O}}$-continuity as well as $\underline{\mathrm{O}}$-continuity.

\section{Fixed point theorems for linear contractions}

In this section, we present sharpened forms of Theorems 1-4, which are new results on their own and are proved without completeness (of the metric space), without continuity (of the underlying mapping) and without the $I C U$ property $/ D C L$ property $/ M C B$ property (of an ordered metric space).

Theorem 5 Let $(X, d, \preceq)$ be an ordered metric space and $f$ a self-mapping on $X$. Suppose that the following conditions hold:

(a) $(X, d, \preceq)$ is $\overline{\mathrm{O}}$-complete,

(b) $f$ is increasing,

(c) either $f$ is $\overline{\mathrm{O}}$-continuous or $(X, d, \preceq)$ has the ICC property,

(d) there exists $x_{0} \in X$ such that $x_{0} \preceq f\left(x_{0}\right)$,

(e) there exists $\alpha \in[0,1)$ such that

$$
d(f x, f y) \leq \alpha d(x, y) \quad \forall x, y \in X \text { with } x \prec \succ y .
$$

Then $f$ has a fixed point.

Proof In view of assumption (d) if $x_{0}=f\left(x_{0}\right)$, then $x_{0}$ is a fixed point of $f$ and hence proof is completed. Otherwise, define a sequence $\left\{x_{n}\right\}$ of Picard iterates, i.e., $x_{n}=f^{n}\left(x_{0}\right) \forall n \in \mathbb{N}_{0}$. As $x_{0} \preceq f\left(x_{0}\right)$ and $f$ is increasing, we obtain by induction that

$$
f\left(x_{0}\right) \preceq f^{2}\left(x_{0}\right) \preceq f^{3}\left(x_{0}\right) \preceq \cdots \preceq f^{n}\left(x_{0}\right) \preceq f^{n+1}\left(x_{0}\right) \preceq \cdots
$$

so that

$$
x_{n} \preceq x_{n+1} \quad \forall n \in \mathbb{N}_{0} .
$$

Thus the sequence $\left\{x_{n}\right\}$ is increasing. Applying the contractivity condition (e) to (1), we deduce, for all $n \in \mathbb{N}$, that

$$
d\left(x_{n}, x_{n+1}\right) \leq \alpha d\left(x_{n-1}, x_{n}\right)
$$

By induction, (2) reduces to

$$
d\left(x_{n}, x_{n+1}\right) \leq \alpha d\left(x_{n-1}, x_{n}\right) \leq \alpha^{2} d\left(x_{n-2}, x_{n-1}\right) \leq \cdots \leq \alpha^{n} d\left(x_{0}, x_{1}\right) \quad \forall n \in \mathbb{N},
$$

so that

$$
d\left(x_{n}, x_{n+1}\right) \leq \alpha^{n} d\left(x_{0}, x_{1}\right) \quad \forall n \in \mathbb{N} .
$$


For $n<m$, using triangular inequality and (3), we obtain

$$
\begin{aligned}
d\left(x_{n}, x_{m}\right) & \leq d\left(x_{n}, x_{n+1}\right)+d\left(x_{n+1}, x_{n+2}\right)+\cdots+d\left(x_{m-1}, x_{m}\right) \\
& \leq\left(\alpha^{n}+\alpha^{n+1}+\cdots+\alpha^{m-1}\right) d\left(x_{0}, x_{1}\right) \\
& =\frac{\alpha^{n}-\alpha^{m}}{1-\alpha} d\left(x_{0}, x_{1}\right) \\
& \leq \frac{\alpha^{n}}{1-\alpha} d\left(x_{0}, x_{1}\right) \\
& \rightarrow 0 \text { as } m, n \rightarrow \infty,
\end{aligned}
$$

which implies that the sequence $\left\{x_{n}\right\}$ is Cauchy in $X$. Therefore, $\left\{x_{n}\right\}$ is an increasing Cauchy sequence. As $X$ is $\overline{\mathrm{O}}$-complete, $\exists x \in X$ such that $x_{n} \stackrel{d}{\longrightarrow} x$, which, on combining with (1), yields

$$
x_{n} \uparrow x
$$

Now, in lieu of (c), first, we assume that $f$ is $\overline{\mathrm{O}}$-continuous. Then using (4) and $\overline{\mathrm{O}}$ continuity of $f$, we obtain $x_{n+1}=f\left(x_{n}\right) \stackrel{d}{\longrightarrow} f(x)$. Owing to the uniqueness of limit, we obtain $f(x)=x$, i.e., $x$ is a fixed point of $f$.

Second, let us assume that $(X, d, \preceq)$ has the ICC property. Then using (4) and the ICC property of $X$, we may obtain a subsequence $\left\{x_{n_{k}}\right\}$ of $\left\{x_{n}\right\}$ such that

$$
x_{n_{k}} \prec \succ x \quad \forall k \in \mathbb{N}_{0} .
$$

Applying the contractivity condition (e) to (5) and using $x_{n_{k}} \stackrel{d}{\longrightarrow} x$, we obtain

$$
\begin{aligned}
d\left(x_{n_{k}+1}, f x\right) & =d\left(f x_{n_{k}}, f x\right) \leq \alpha d\left(x_{n_{k}}, x\right) \\
& \rightarrow 0 \quad \text { as } k \rightarrow \infty
\end{aligned}
$$

so that $x_{n_{k}+1} \stackrel{d}{\longrightarrow} f(x)$. Again, owing to the uniqueness of the limit, we obtain $f(x)=x$ so that $x$ is a fixed point of $f$.

Next, we present a dual result to Theorem 5 .

Theorem 6 Theorem 5 remains true if certain involved terms namely: $\overline{\mathrm{O}}$-complete, $\overline{\mathrm{O}}$ continuous and the ICC property are, respectively, replaced by $\underline{\mathrm{O}}$-complete, $\underline{\mathrm{O}}$-continuous and the DCC property provided the assumption (d) is replaced by the following (besides retaining the rest):

(d)' there exists $x_{0} \in X$ such that $x_{0} \succeq f\left(x_{0}\right)$.

Proof The scheme of the proof is similar to the one followed in the proof of Theorem 5 . Following the lines of the proof of Theorem 5 , using $x_{0} \succeq f\left(x_{0}\right)$ and the increasing property of $f$, we obtain by induction

$$
f\left(x_{0}\right) \succeq f^{2}\left(x_{0}\right) \succeq f^{3}\left(x_{0}\right) \succeq \cdots \succeq f^{n}\left(x_{0}\right) \succeq f^{n+1}\left(x_{0}\right) \succeq \cdots
$$


so that

$$
x_{n} \succeq x_{n+1} \quad \forall n \in \mathbb{N}_{0} .
$$

i.e. the sequence $\left\{x_{n}\right\}$ is decreasing. An analogous procedure to the proof of Theorem 5, we can also show that $\left\{x_{n}\right\}$ is Cauchy. The $\underline{\mathrm{O}}$-completeness of $X$ implies the existence of $x \in X$ such that

$$
x_{n} \downarrow x .
$$

Now, in lieu of (c), first, we assume that $f$ is $\underline{\mathrm{O}}$-continuous. Then using (6) and $\underline{\mathrm{O}}$ continuity of $f$, we obtain $x_{n+1}=f\left(x_{n}\right) \stackrel{d}{\longrightarrow} f(x)$. Owing to the uniqueness of limit, we obtain $f(x)=x$, i.e., $x$ is a fixed point of $f$.

Second, let us assume that $(X, d, \preceq)$ has the $D C C$ property. Then using (6) and the $D C C$ property of $X$, we may obtain a subsequence $\left\{x_{n_{k}}\right\}$ of $\left\{x_{n}\right\}$ such that

$$
x_{n_{k}} \prec \succ x \quad \forall k \in \mathbb{N}_{0} .
$$

Applying the contractivity condition (e) to (7) and using $x_{n_{k}} \stackrel{d}{\longrightarrow} x$, we obtain

$$
\begin{aligned}
d\left(x_{n_{k}+1}, f x\right) & =d\left(f x_{n_{k}}, f x\right) \leq \alpha d\left(x_{n_{k}}, x\right) \\
& \rightarrow 0 \quad \text { as } k \rightarrow \infty
\end{aligned}
$$

so that $x_{n_{k}+1} \stackrel{d}{\longrightarrow} f(x)$. Again, owing to the uniqueness of limit, we obtain $f(x)=x$ so that $x$ is a fixed point of $f$.

Remark 4 Notice that Theorems 5 and 6 sharpen Theorems 1 and 2, respectively. Here we observe that in Nieto and Rodríguez-López theorems the completeness, continuity, the $I C U$ property, and the $D C L$ property are not necessary as the same can alternately be replaced by their respective relatively weaker notions.

On combining Theorem 5 and Theorem 6, we obtain the following result.

Theorem 7 Theorem 5 remains true if certain involved terms namely: $\overline{\mathrm{O}}$-complete, $\overline{\mathrm{O}}$ continuous, and the ICC property are, respectively, replaced by $\mathrm{O}$-complete, O-continuous and the MCC property provided the assumption (d) is replaced by the following (besides retaining the rest):

(d)" there exists $x_{0} \in X$ such that $x_{0} \prec \succ f\left(x_{0}\right)$.

Finally, we prove certain unique fixed point results corresponding to Theorems 5-7.

Theorem 8 In addition to the hypotheses of Theorem 5 (resp. Theorem 6 or Theorem 7), suppose that the following condition holds:

(f) $\mathrm{C}(f x, f y, \prec \succ)$ is nonempty for each $x, y \in X$.

Then $f$ has a unique fixed point. 
Proof In view of Theorem 5 (resp. Theorem 6 or Theorem 7), the set of fixed points of $f$ is nonempty. Take two fixed points $x$ and $y$ of $f$, i.e.,

$$
f(x)=x \text { and } f(y)=y .
$$

By assumption (f), there exists a $\prec \succ$-chain (say $\left.\left\{z_{1}, z_{2}, \ldots, z_{k}\right\}\right)$ between $f(x)$ and $f(y)$ so that

$$
z_{1}=x, \quad z_{k}=y \quad \text { and } \quad z_{i} \prec \succ z_{i+1} \quad \text { for each } i(1 \leq i \leq k-1) .
$$

As $f$ is increasing, we have

$$
f^{n}\left(z_{i}\right) \prec \succ f^{n}\left(z_{i+1}\right) \quad \text { for each } i(1 \leq i \leq k-1) \text { and for each } n \in \mathbb{N}_{0} \text {. }
$$

Making use of (8), (9), (10), the triangular inequality, and assumption (e), we obtain

$$
\begin{aligned}
d(x, y) & =d\left(f^{n} z_{1}, f^{n} z_{k}\right) \leq \sum_{i=1}^{k-1} d\left(f^{n} z_{i}, f^{n} z_{i+1}\right) \leq \alpha \sum_{i=1}^{k-1} d\left(f^{n-1} z_{i}, f^{n-1} z_{i+1}\right) \\
& \leq \alpha^{2} \sum_{i=1}^{k-1} d\left(f^{n-2} z_{i}, f^{n-2} z_{i+1}\right) \leq \cdots \leq \alpha^{n} \sum_{i=1}^{k-1} d\left(z_{i}, z_{i+1}\right) \\
& \rightarrow 0 \quad \text { as } n \rightarrow \infty
\end{aligned}
$$

so that $x=y$. Hence $f$ has a unique fixed point.

\section{Fixed point theorems for nonlinear contractions}

In this section, we discuss some variants of preceding results for nonlinear contractions due to Boyd and Wong [15]. As per standard practice, a function $\varphi:[0, \infty) \rightarrow[0, \infty)$ satisfying $\varphi(t)<t$ for each $t>0$ is called a control function. Further, a self-mapping $f$ defined on a metric space $(X, d)$ is called a nonlinear contraction with respect to control function $\varphi$ (or, in short, $\varphi$-contraction) if

$$
d(f x, f y) \leq \varphi(d(x, y)) \quad \forall x, y \in X
$$

Indeed for each $\alpha \in[0,1)$, on setting $\varphi(t)=\alpha t, \varphi$-contraction reduces to $\alpha$-contraction (i.e. usual contraction). In 1969, Boyd and Wong [15] introduced the following family of control functions:

$$
\Omega=\left\{\varphi:[0, \infty) \rightarrow[0, \infty): \varphi(t)<t \text { for each } t>0 \text { and } \limsup _{r \rightarrow t^{+}} \varphi(r)<t \text { for each } t>0\right\} .
$$

We need the following known results in the proof of our main results of this section.

Lemma 1 [9] Let $\varphi \in \Omega$. If $\left\{a_{n}\right\} \subset(0, \infty)$ is a sequence such that $a_{n+1} \leq \varphi\left(a_{n}\right) \forall n \in \mathbb{N}_{0}$, then $\lim _{n \rightarrow \infty} a_{n}=0$. 
Lemma 2 [12] Let $(X, d)$ be a metric space and $\left\{x_{n}\right\}$ a sequence in $X$ such that $\lim _{n \rightarrow \infty} d\left(x_{n}\right.$, $\left.x_{n+1}\right)=0$. If $\left\{x_{n}\right\}$ is not a Cauchy sequence, then there exist $\epsilon>0$ and two subsequences $\left\{x_{n_{k}}\right\}$ and $\left\{x_{m_{k}}\right\}$ of $\left\{x_{n}\right\}$ such that

(i) $n_{k}>m_{k} \geq k$

(ii) $d\left(x_{m_{k}}, x_{n_{k}}\right) \geq \epsilon$,

(iii) $d\left(x_{m_{k}}, x_{n_{k}-1}\right)<\epsilon$,

(iv) the following four sequences tend to $\epsilon$ when $k \rightarrow \infty$ :

$$
d\left(x_{m_{k}}, x_{n_{k}}\right), d\left(x_{m_{k}+1}, x_{n_{k}}\right), d\left(x_{m_{k}}, x_{n_{k}+1}\right), d\left(x_{m_{k}+1}, x_{n_{k}+1}\right) .
$$

Now, we extend Theorems 5 and 6 for nonlinear contractions as follows.

Theorem 9 Theorem 5 (resp. Theorem 6 or Theorem 7) remains true if the assumption (e) is replaced by the following (besides retaining the rest):

(e)' there exists $\varphi \in \Omega$ such that

$$
d(f x, f y) \leq \varphi(d(x, y)) \quad \forall x, y \in X \text { with } x \prec \succ y .
$$

Proof We start the proof proceeding the lines similar to the proof of Theorem 5 (resp. Theorem 6 or Theorem 7). Following its lines, we define the sequence $\left\{x_{n}\right\}$ of Picard iterates and then we can prove that the sequence $\left\{x_{n}\right\}$ is increasing (resp. decreasing or monotone).

If $x_{n_{0}}=x_{n_{0}+1}$ for some $n_{0} \in \mathbb{N}$, then we have $x_{n_{0}}=f\left(x_{n_{0}}\right)$, i.e., $x_{n_{0}}$ is a fixed point of $f$ so that we are done. On the other hand, if $x_{n} \neq x_{n+1}$ for each $n \in \mathbb{N}_{0}$, then in this case, as an analog of (2), we obtain, for all $n \in \mathbb{N}$,

$$
d\left(x_{n}, x_{n+1}\right) \leq \varphi\left(d\left(x_{n-1}, x_{n}\right)\right)
$$

which on applying Lemma 1 gives rise

$$
\lim _{n \rightarrow \infty} d\left(x_{n}, x_{n+1}\right)=0
$$

Next, we show that $\left\{x_{n}\right\}$ is a Cauchy sequence. On the contrary, suppose that $\left\{x_{n}\right\}$ is not a Cauchy sequence. Hence, in view of (11) and Lemma 2, there exist $\epsilon>0$ and two subsequences $\left\{x_{n_{k}}\right\}$ and $\left\{x_{m_{k}}\right\}$ of $\left\{x_{n}\right\}$ such that $n_{k}>m_{k} \geq k, d\left(x_{m_{k}}, x_{n_{k}}\right) \geq \epsilon, d\left(x_{m_{k}}, x_{n_{k}-1}\right)<\epsilon$, and

$$
\begin{aligned}
\lim _{k \rightarrow \infty} d\left(x_{m_{k}}, x_{n_{k}}\right) & =\lim _{k \rightarrow \infty} d\left(x_{m_{k}+1}, x_{n_{k}}\right) \\
& =\lim _{k \rightarrow \infty} d\left(x_{m_{k}}, x_{n_{k}+1}\right) \\
& =\lim _{k \rightarrow \infty} d\left(x_{m_{k}+1}, x_{n_{k}+1}\right)=\epsilon .
\end{aligned}
$$

Denote $r_{k}:=d\left(x_{m_{k}}, x_{n_{k}}\right)$. As $m_{k}<n_{k}$, due to (1) we have $x_{m_{k}} \preceq x_{n_{k}}$. On applying the contractivity condition $(e)^{\prime}$, we obtain

$$
d\left(x_{m_{k}+1}, x_{n_{k}+1}\right)=d\left(f x_{m_{k}}, f x_{n_{k}}\right) \leq \varphi\left(d\left(x_{m_{k}}, x_{n_{k}}\right)\right)=\varphi\left(r_{k}\right)
$$


so that

$$
d\left(x_{m_{k}+1}, x_{n_{k}+1}\right) \leq \varphi\left(r_{k}\right) .
$$

On taking the limit superior as $k \rightarrow \infty$ in (13) and using (12) and the definition of $\Omega$, we have

$$
\epsilon=\limsup _{k \rightarrow \infty} d\left(x_{m_{k}+1}, x_{n_{k}+1}\right) \leq \limsup _{k \rightarrow \infty} \varphi\left(r_{k}\right)=\limsup _{r_{k} \rightarrow \epsilon^{+}} \varphi\left(r_{k}\right)<\epsilon,
$$

which is a contradiction. Therefore $\left\{x_{n}\right\}$ is a Cauchy sequence.

Now, assumption (a) implies the existence of $x \in X$ such that $x_{n} \uparrow x$ (resp. $x_{n} \downarrow x$ or $x_{n} \uparrow \downarrow x$ ). To prove $x \in X$ is a fixed point of $f$, firstly we suppose that $f$ is $\overline{\mathrm{O}}$-continuous (resp. O-continuous or O-continuous). In this case, proceeding along the lines of the proof of Theorem 5 (resp. Theorem 6 or Theorem 7 ), we can show that $f(x)=x$. Otherwise suppose that $(X, d, \preceq)$ has the $I C C$ property (resp. the $D C C$ property or the $M C C$ property), which provides the existence of a subsequence $\left\{x_{n_{k}}\right\}$ of $\left\{x_{n}\right\}$ such that

$$
x_{n_{k}} \prec \succ x \quad \forall k \in \mathbb{N}_{0} .
$$

On using (14) and assumption $(e)^{\prime}$, we obtain

$$
d\left(x_{n_{k}+1}, f x\right)=d\left(f x_{n_{k}}, f x\right) \leq \varphi\left(d\left(x_{n_{k}}, x\right)\right) \quad \forall k \in \mathbb{N}_{0} .
$$

We claim that

$$
d\left(x_{n_{k}+1}, f x\right) \leq d\left(x_{n_{k}}, x\right) \quad \forall k \in \mathbb{N} .
$$

On account of two different possibilities arising here, we consider a partition $\left\{\mathbb{N}^{0}, \mathbb{N}^{+}\right\}$of $\mathbb{N}$, i.e., $\mathbb{N}^{0} \cup \mathbb{N}^{+}=\mathbb{N}$ and $\mathbb{N}^{0} \cap \mathbb{N}^{+}=\emptyset$ verifying that

(i) $d\left(x_{n_{k}}, x\right)=0 \forall k \in \mathbb{N}^{0}$,

(ii) $d\left(x_{n_{k}}, x\right)>0 \forall k \in \mathbb{N}^{+}$.

In case (i), we have $d\left(f x_{n_{k}}, f x\right)=0 \forall k \in \mathbb{N}^{0}$, which implies that $d\left(x_{n_{k}+1}, f x\right)=0 \forall k \in \mathbb{N}^{0}$ and hence (15) holds for all $k \in \mathbb{N}^{0}$. If case (ii) holds, by the definition of $\Omega$, we have $d\left(x_{n_{k}+1}, f x\right) \leq \varphi\left(d\left(x_{n_{k}}, x\right)\right)<d\left(x_{n_{k}}, x\right) \forall k \in \mathbb{N}^{+}$and hence (15) holds for all $k \in \mathbb{N}^{+}$. Thus (15) holds for all $k \in \mathbb{N}$.

Taking the limit of (15) as $n \rightarrow \infty$ and using $x_{n_{k}} \stackrel{d}{\longrightarrow} x$, we obtain $x_{n_{k}+1} \stackrel{d}{\longrightarrow} f(x)$. Owing to the uniqueness of limit, we obtain $f(x)=x$ so that $x$ is a fixed point of $f$.

Remark 5 Notice that for $\varphi(t)=\alpha \cdot t$ with $\alpha \in[0,1)$, Theorem 9 reduces to Theorems 5-7.

Theorem 10 In addition to the hypotheses of Theorem 9, suppose that the following assumption (f) (of Theorem 8) holds, then $f$ has a unique fixed point.

Proof We can prove this result easily by using the similar technique as utilized in Theorem 8. 


\section{Application to boundary value problem}

In this section, we present an example, where Theorems 9 and 10 can be applied. We study the existence and uniqueness of solution for the following first order periodic boundary value problem:

$$
\left\{\begin{array}{l}
u^{\prime}(t)=f(t, u(t)), \quad t \in I=[0, T] \\
u(0)=u(T)
\end{array}\right.
$$

where $T>0$ and $f: I \times \mathbb{R} \rightarrow \mathbb{R}$ is a continuous function.

Let $\mathcal{C}(I)$ denote the space of all continuous functions defined on $I$. Now, we recall the following definitions.

Definition 7 [5] A function $\alpha \in \mathcal{C}^{1}(I)$ is called a lower solution of (16), if

$$
\left\{\begin{array}{l}
\alpha^{\prime}(t) \leq f(t, \alpha(t)), \quad t \in I, \\
\alpha(0) \leq \alpha(T)
\end{array}\right.
$$

Definition 8 [5] A function $\alpha \in \mathcal{C}^{1}(I)$ is called an upper solution of (16), if

$$
\left\{\begin{array}{l}
\alpha^{\prime}(t) \geq f(t, \alpha(t)), \quad t \in I, \\
\alpha(0) \geq \alpha(T) .
\end{array}\right.
$$

Let $\mathfrak{F}$ denote the family of functions $\phi:[0, \infty] \rightarrow[0, \infty]$ satisfying the following conditions:

(i) $\phi$ is continuous and increasing,

(ii) $\phi(t)<t$ for each $t>0$.

Typical examples of $\mathfrak{F}$ are $\phi(t)=\alpha \cdot t, 0 \leq \alpha<1, \phi(t)=\frac{t}{1+t}$, and $\phi(t)=\ln (1+t)$. Also, clearly $\mathfrak{F} \subset \Omega$.

Now, we prove the following result regarding the existence and uniqueness of a solution of problem (16) in the presence of a lower solution or an upper solution.

Theorem 11 In addition to the problem (16), suppose that there exist $\lambda>0$ and $\phi \in \mathfrak{F}$ such that for all $x, y \in \mathbb{R}$ with $x \leq y$

$$
0 \leq f(t, y)+\lambda y-[f(t, x)+\lambda x] \leq \lambda \phi(y-x) .
$$

Then the existence of a lower solution or an upper solution of problem (16) provides the existence and uniqueness of a solution of problem (16).

Proof Problem (16) can be rewritten as

$$
\left\{\begin{array}{l}
u^{\prime}(t)+\lambda u(t)=f(t, u(t))+\lambda u(t), \quad \forall t \in I, \\
u(0)=u(T) .
\end{array}\right.
$$


Problem (18) is equivalent to the integral equation

$$
u(t)=\int_{0}^{T} G(t, \xi)[f(\xi, u(\xi))+\lambda u(\xi)] d \xi
$$

where the Green function $G(t, \xi)$ is given by

$$
G(t, \xi)= \begin{cases}\frac{e^{\lambda(T+\xi-t)}}{e^{\lambda T}-1}, & 0 \leq \xi<t \leq T, \\ \frac{e^{\lambda(\xi-t)}}{e^{\lambda T}-1}, & 0 \leq t<\xi \leq T .\end{cases}
$$

Define a function $\mathcal{A}: \mathcal{C}(I) \rightarrow \mathcal{C}(I)$ by

$$
(\mathcal{A} u)(t)=\int_{0}^{T} G(t, \xi)[f(\xi, u(\xi))+\lambda u(\xi)] d \xi \quad \forall t \in I .
$$

Clearly, if $u \in \mathcal{C}(I)$ is a fixed point of $\mathcal{A}$ then $u \in \mathcal{C}^{1}(I)$ is a solution of (19) and hence of (16).

On $\mathcal{C}(I)$, define a metric $d$ given by

$$
d(u, v)=\sup _{t \in I}|u(t)-v(t)| \quad \forall u, v \in \mathcal{C}(I)
$$

On $\mathcal{C}(I)$, define a partial order $\preceq$ given by

$$
u, v \in \mathcal{C}(I) ; \quad u \preceq v \quad \Longleftrightarrow u(t) \leq v(t) \quad \forall t \in I .
$$

Now we check that all the conditions of Theorem 9 are satisfied:

(a) Clearly, $(\mathcal{C}(I), d, \preceq)$ is an O-complete ordered metric space.

(b) Take $u, v \in \mathcal{C}(I)$ such that $u \preceq v$, then by (17), we obtain

$$
f(t, u(t))+\lambda u(t) \leq f(t, v(t))+\lambda v(t) \quad \forall t \in I
$$

On using (20), (23), and the fact that $G(t, \xi)>0$ for $(t, \xi) \in I \times I$, we get

$$
\begin{aligned}
(\mathcal{A} u)(t) & =\int_{0}^{T} G(t, \xi)[f(\xi, u(\xi))+\lambda u(\xi)] d \xi \\
& \leq \int_{0}^{T} G(t, \xi)[f(\xi, \nu(\xi))+\lambda v(\xi)] d \xi \\
& =(\mathcal{A} v)(t) \quad \forall t \in I,
\end{aligned}
$$

which owing to (22) implies that $\mathcal{A}(u) \preceq \mathcal{A}(v)$ so that $\mathcal{A}$ is increasing.

(c) Take a sequence $\left\{u_{n}\right\} \subset \mathcal{C}(I)$ such that $u_{n} \uparrow \downarrow u \in \mathcal{C}(I)$, then for each $t \in I,\left\{u_{n}(t)\right\}$ is monotone sequence in $\mathbb{R}$ converging to $u(t)$. Hence, for all $n \in \mathbb{N}_{0}$ and for all $t \in I$, we have

$$
\begin{array}{ll}
u_{n}(t) \leq u(t) & \text { if }\left\{u_{n}(t)\right\} \text { is increasing, } \\
u_{n}(t) \geq u(t) & \text { if }\left\{u_{n}(t)\right\} \text { is decreasing, }
\end{array}
$$

which by using (22) implies that $u_{n} \prec \succ u \forall n \in \mathbb{N}_{0}$ so that $(\mathcal{C}(I), d, \preceq)$ has the $M C C$ property. 
(d) Let $\alpha \in \mathcal{C}^{1}(I)$ be a lower solution of (16), then we have

$$
\alpha^{\prime}(t)+\lambda \alpha(t) \leq f(t, \alpha(t))+\lambda \alpha(t) \quad \forall t \in I
$$

Multiplying on both sides by $e^{\lambda t}$, we get

$$
\left(\alpha(t) e^{\lambda t}\right)^{\prime} \leq[f(t, \alpha(t))+\lambda \alpha(t)] e^{\lambda t} \quad \forall t \in I
$$

which implies that

$$
\alpha(t) e^{\lambda t} \leq \alpha(0)+\int_{0}^{t}[f(\xi, \alpha(\xi))+\lambda \alpha(\xi)] e^{\lambda \xi} d \xi \quad \forall t \in I .
$$

As $\alpha(0) \leq \alpha(T)$, we get

$$
\alpha(0) e^{\lambda T} \leq \alpha(T) e^{\lambda T} \leq \alpha(0)+\int_{0}^{T}[f(\xi, \alpha(\xi))+\lambda \alpha(\xi)] e^{\lambda \xi} d \xi
$$

so that

$$
\alpha(0) \leq \int_{0}^{T} \frac{e^{\lambda \xi}}{e^{\lambda T}-1}[f(\xi, \alpha(\xi))+\lambda \alpha(\xi)] d \xi .
$$

On using (24) and (25), we obtain

$$
\begin{aligned}
\alpha(t) e^{\lambda t} & \leq \int_{0}^{T} \frac{e^{\lambda \xi}}{e^{\lambda T}-1}[f(\xi, \alpha(\xi))+\lambda \alpha(\xi)] d \xi+\int_{0}^{t} e^{\lambda \xi}[f(\xi, \alpha(\xi))+\lambda \alpha(\xi)] d \xi \\
& =\int_{0}^{t} \frac{e^{\lambda(T+\xi)}}{e^{\lambda T}-1}[f(\xi, \alpha(\xi))+\lambda \alpha(\xi)] d \xi+\int_{t}^{T} \frac{e^{\lambda \xi}}{e^{\lambda T}-1}[f(\xi, \alpha(\xi))+\lambda \alpha(\xi)] d \xi
\end{aligned}
$$

so that

$$
\begin{aligned}
\alpha(t) & \leq \int_{0}^{t} \frac{e^{\lambda(T+\xi-t)}}{e^{\lambda T}-1}[f(\xi, \alpha(\xi))+\lambda \alpha(\xi)] d \xi+\int_{t}^{T} \frac{e^{\lambda(\xi-t)}}{e^{\lambda T}-1}[f(\xi, \alpha(\xi))+\lambda \alpha(\xi)] d \xi \\
& =\int_{0}^{T} G(t, \xi)[f(\xi, \alpha(\xi))+\lambda \alpha(\xi)] d \xi \\
& =(\mathcal{A} \alpha)(t)
\end{aligned}
$$

for all $t \in I$, which implies that $\alpha \preceq \mathcal{A}(\alpha)$. Otherwise, if $\alpha \in \mathcal{C}^{1}(I)$ is an upper solution of (16), then in a similar manner, we get $\alpha \succeq \mathcal{A}(\alpha)$. Hence, in both the cases, we have $\alpha \prec \succ \mathcal{A}(\alpha)$, for some lower or upper solution $\alpha$.

(e)' Take $u, v \in \mathcal{C}(I)$ such that $u \preceq v$, using (17), (20), and (21), we obtain

$$
\begin{aligned}
d(\mathcal{A} u, \mathcal{A} v) & =\sup _{t \in I}|(\mathcal{A} u)(t)-(\mathcal{A} v)(t)|=\sup _{t \in I}((\mathcal{A} v)(t)-(\mathcal{A} u)(t)) \\
& \leq \sup _{t \in I} \int_{0}^{T} G(t, \xi)[f(\xi, v(\xi))+\lambda v(\xi)-f(\xi, u(\xi))-\lambda u(\xi)] d \xi \\
& \leq \sup _{t \in I} \int_{0}^{T} G(t, \xi) \lambda \phi(v(\xi)-u(\xi)) d \xi .
\end{aligned}
$$


Given that $\phi$ is increasing on $[0, \infty)$ and $u \preceq v$, which implies that $\phi(v(\xi)-u(\xi)) \leq$ $\phi(d(u, v))$. Hence, $(26)$ reduces to

$$
\begin{aligned}
d(\mathcal{A} u, \mathcal{A} v) & \leq \lambda \phi(d(u, v)) \sup _{t \in I} \int_{0}^{T} G(t, \xi) d \xi \\
& =\lambda \phi(d(u, v)) \sup _{t \in I} \frac{1}{e^{\lambda T}-1}\left(\left.\frac{1}{\lambda} e^{\lambda(T+\xi-t)}\right|_{0} ^{t}+\left.\frac{1}{\lambda} e^{\lambda(\xi-t)}\right|_{t} ^{T}\right) \\
& =\lambda \phi(d(u, v)) \frac{1}{\lambda\left(e^{\lambda T}-1\right)}\left(e^{\lambda T}-1\right) \\
& =\phi(d(u, v))
\end{aligned}
$$

so that

$$
d(\mathcal{A} u, \mathcal{A} v) \leq \phi(d(u, v)) \quad \forall u, v \in \mathcal{C}(I) \text { such that } u \preceq v
$$

where $\phi \in \mathfrak{F} \subset \Omega$.

Hence, all the conditions of Theorem 9 are satisfied consequently $\mathcal{A}$ has a fixed point.

Finally choose arbitrary $u, v \in \mathcal{C}(I)$, then $w:=\max \{\mathcal{A} u, \mathcal{A} v\} \in \mathcal{C}(I)$. As $\mathcal{A}(u) \preceq w$ and $\mathcal{A}(v) \preceq w,\{\mathcal{A} u, w, \mathcal{A} v\}$ is a $\prec \succ$-chain between $\mathcal{A}(u)$ and $\mathcal{A}(v)$. Thus, by Theorem $10, \mathcal{A}$ has a unique fixed point, which is, indeed, a unique solution of problem (16).

\section{Competing interests}

All the authors declare that there is no conflict of interests.

\section{Authors' contributions}

All authors contributed equally to the writing of this paper. All authors read and approved the final manuscript.

\section{Author details}

'Department of Mathematics, King Abdulaziz University, P.O. Box 80203, Jeddah, 21589, Saudi Arabia. ${ }^{2}$ Department of Mathematics, Aligarh Muslim University, Aligarh, Uttar Pradesh 202002, India.

\section{Acknowledgements}

The first author gratefully acknowledges the support from the Deanship of Scientific Research (DSR) at King Abdulaziz University (KAU) in Jeddah, Kingdom of Saudi Arabia during this research. All the authors are thankful to two anonymous learned referees for their encouraging comments.

Received: 23 June 2015 Accepted: 20 October 2015 Published online: 02 November 2015

\section{References}

1. O'Regan, D, Petruşel, A: Fixed point theorems for generalized contractions in ordered metric spaces. J. Math. Anal. Appl. 341(2), 1241-1252 (2008)

2. Turinici, M: Abstract comparison principles and multivariable Gronwall-Bellman inequalities. J. Math. Anal. Appl. 117(1), 100-127 (1986)

3. Turinici, M: Fixed points for monotone iteratively local contractions. Demonstr. Math. 19(1), 171-180 (1986)

4. Ran, ACM, Reurings, MCB: A fixed point theorem in partially ordered sets and some applications to matrix equations. Proc. Am. Math. Soc. 132(5), 1435-1443 (2004)

5. Nieto, JJ, Rodríguez-López, R: Contractive mapping theorems in partially ordered sets and applications to ordinary differential equations. Order 22(3), 223-239 (2005)

6. Agarwal, RP, El-Gebeily, MA, O'Regan, D: Generalized contractions in partially ordered metric spaces. Appl. Anal. 87(1), 109-116 (2008)

7. Altun, I, Simsek, H: Some fixed point theorems on ordered metric spaces and application. Fixed Point Theory Appl. $2010,621469(2010)$

8. Nashine, HK, Altun, I: Fixed point theorems for a pair of mappings satisfying a generalized weakly contractive condition in ordered metric spaces. Hacet. J. Math. Stat. 41(2), 243-253 (2012)

9. Alam, A, Khan, AR, Imdad, M: Some coincidence theorems for generalized nonlinear contractions in ordered metric spaces with applications. Fixed Point Theory Appl. 2014, 216 (2014)

10. Alam, A, Khan, QH, Imdad, M: Enriching the recent coincidence theorems for nonlinear contractions in ordered metric spaces. Fixed Point Theory Appl. 2015, 141 (2015)

11. Gnana Bhaskar, T, Lakshmikantham, V: Fixed point theorems in partially ordered metric spaces and applications. Nonlinear Anal. 65(7), 1379-1393 (2006) 
12. Jleli, M, Rajic, VC, Samet, B, Vetro, C: Fixed point theorems on ordered metric spaces and applications to nonlinear elastic beam equations. J. Fixed Point Theory Appl. 12, 175-192 (2012)

13. Turinici, M: Nieto-Lopez theorems in ordered metric spaces. Math. Stud. 81(1-4), 219-229 (2012)

14. Turinici, M: Linear contractions in product ordered metric spaces. Ann. Univ. Ferrara 59, 187-198 (2013)

15. Boyd, DW, Wong, JSW: On nonlinear contractions. Proc. Am. Math. Soc. 20, 458-464 (1969)

Submit your manuscript to a SpringerOpen ${ }^{\circ}$ journal and benefit from:

- Convenient online submission

Rigorous peer review

- Immediate publication on acceptance

- Open access: articles freely available online

- High visibility within the field

- Retaining the copyright to your article 